\title{
Simulation of ground support performance in highly fractured and bulked rock masses with advanced 3DEC bolt model
}

\author{
L Bouzeran Itasca Consulting Group Inc., USA \\ J Furtney Itasca Consulting Group Inc., USA \\ M Pierce Pierce Engineering, USA \\ J Hazzard Itasca Consulting Group Inc., USA \\ JV Lemos Itasca Consulting Group Inc., USA
}

\begin{abstract}
The design of effective ground support is critical to the success of the next generation of large cave mines. Typically, the behaviour of rock in this setting is controlled by shear and opening movements along fractures induced by the excavation and cycles of loading and unloading during mine development and operation. Support of highly stressed fractured rock shows two important aspects: (i) in blocky or fractured rock the majority of the support deformation is localised at fractures or joints and (ii) the resistance to fracture shear displacement offered by support is important. These observations have led to the use of the discrete element method based three-dimensional bonded block model (BBM) to represent the rock and the hybrid bolt model to represent the bolt support. The hybrid bolt model is an improvement on the classical cable bolt model, which features a more realistic resistance to fracture shear displacement and allows bolt installation in a fractured rock mass exhibiting open joints. This paper presents the application of the BBM and hybrid-bolt numerical model to study the performance of tunnelling at depth under a caving-induced stress path. The effect of support pressure, bolts presence, and partial debonding of bolts, is explored. The influence of rock mass quality and support installation timing on the support efficiency is also investigated. It has been found that a small surface pressure is critical for tunnel performance. When a rock mass experiences large deformation and joint opening, local axial and shearing straining of bolts at joints intersection become important and can lead to rupture. Intense axial straining can be mitigated through debonding and intense shearing can be reduced through the use of bolts with higher shear resistance, such as rebars.
\end{abstract}

Keywords: tunnel support bolt, numerical simulation, bulking, 3DEC

\section{$1 \quad$ Introduction}

Massive underground mining is becoming ever more prevalent, specifically caving mines at depths greater than $1,000 \mathrm{~m}$. Tunnel support designs must consider the development process of cave mine infrastructure in deep, highly stressed environments. Mine development sequencing and cave advance affect the re-distribution of induced stresses on mine infrastructure. Support designs must consider the excavation instability problems resulting from associated loading and unloading. Garza-Cruz and Pierce (2014) have shown that the discrete element method (DEM) is preferable to continuum approaches for representing the spalling and bulking that occur at depth. Discontinuum approaches, such as DEM, can explicitly represent the initiation and propagation of fractures. DEM models are able to show that brittle failure is dominant at low confinement whilst brittle failure and the propagation of tensile fractures is inhibited at higher confinement. This mechanism is important for accurate stability assessment and support design.

3DEC (Itasca Consulting Group, Inc. 2013) allows for the construction of synthetic rock samples with zero initial porosity. The rock mass model consists of a collection of interlocked tetrahedral blocks bonded at their contacts. The tensile strength of these contact bonds is heterogeneous. A bond breaks when the bond tensile or shear strength is exceeded. After a contact is broken, the contact resists joint shear motion via 
Coulomb friction. The contacts represent a network of low persistence veins and open fractures while the blocks themselves represent unveined intact rock. Such models are referred to as bonded block models (BBM). A rockbolt model was recently added to 3DEC version 5.2 which enables the study of support effectiveness using the BBM approach in this setting. This was challenging for two reasons. First, the mechanical behaviour of bolted rock fractures is very complex and not fully described in the literature despite significant research. A numerical implementation has to be simple enough to be computationally manageable in a DEM simulation at the tunnel scale. The implementation must also reproduce the key components of the reinforcing effect of the bolt using a reasonable number of input parameters. Furthermore, in a BBM, support has to be installed in an already open jointed/fractured rock mass due to the partial relaxation of the tunnel at the time of the support installation. A new structural element developed in 3DEC called the 'hybrid bolt' is able to reproduce both the shear and axial behaviour of rockbolts, such as fully grouted rebar, cable bolts and split sets. The model is a combination of a traditional cable element to model the axial behaviour and local shear spring at fracture intersections to model the shear resistance induced by the presence of the bolt system (steel and grout). Both elements can yield and rupture and can reproduce the three stages of load response (elastic, yield and plastic stages) typically observed in laboratory tests. A literature review and details of the implementation and calibration of the hybrid bolt model have been presented in detailed in Bouzeran et al. (2016).

The present paper focuses on the application of this new model to study tunnel performance at depth. Section 2 describes the tunnel model and the support design, which includes cable bolts and grouted rebars. Section 3 gives a detailed outline of the different simulations and corresponding sequences; an unsupported tunnel, along with partially debonded and bonded cables support systems are considered. Finally, Section 4 presents the main results of the simulations.

\section{Tunnel model description}

The model is two-and-a-half dimensional (2.5D); it is a slice of tunnel $2.4 \mathrm{~m}$ long, as shown in Figure 1 . The BBM sample is embedded in an elastic boundary and the model was equilibrated under an in situ stress state equivalent to a tunnel depth of $1,200 \mathrm{~m}\left(\sigma_{v}=31 \mathrm{MPa}, \sigma_{H}=3 \cdot \sigma_{v}=93 \mathrm{MPa}\right.$, and $\left.\sigma_{\mathrm{h}}=1.6 \cdot \sigma_{\mathrm{v}}=50 \mathrm{MPa}\right)$. The Young's modulus of the elastic boundary is set to match that of the rock mass ( $11 \mathrm{GPa}$ ), and Poisson's ratio is assumed to be 0.25 . A tunnel aligned with the direction of $\sigma_{h}$ was mined through the 3DEC BBM material, with dimensions shown in Figure 1 . Symmetry boundary conditions are used at all vertical boundaries of the model to simulate an array of parallel tunnels at $14 \mathrm{~m}$ intervals (centre to centre). Two sets of rock mass properties have been studied. The first set is considered equivalent to a 'medium' quality rock mass, and referred as 'medium rock mass' in the following paragraphs. A lower quality rock mass has been defined by lowering the strength of the medium rock mass; it is referred to as 'weak rock mass' in the following paragraphs. Table 1 summarises the properties of both medium and weak rock masses used for the simulations.

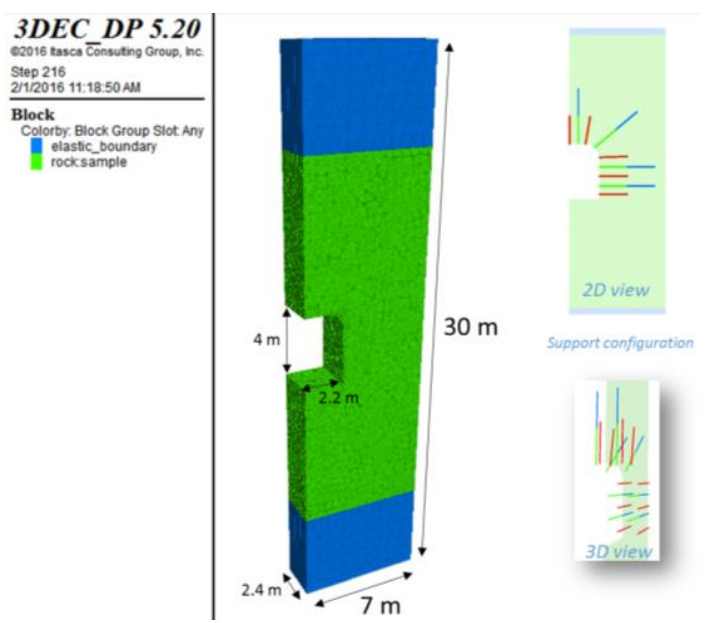

Figure 1 Model geometry (BBM model in green and elastic boundary in blue) 
Table 1 Elastic block and contact mechanical properties of the medium and weak rock masses

\begin{tabular}{ll}
\hline Block properties & \\
\hline Young's modulus & $50 \mathrm{GPa}$ \\
\hline Poisson's ratio & 0.25 \\
\hline Density & $2,650 \mathrm{~kg} / \mathrm{m}^{3}$ \\
\hline Contact properties & \\
\hline Normal stiffness & $105 \mathrm{GPa} / \mathrm{m}$ \\
\hline Shear stiffness & $52.5 \mathrm{GPa} / \mathrm{m}$ \\
\hline Peak friction angle & $35^{\circ}$ (medium) or 30 ${ }^{\circ}$ (weak) \\
\hline Residual friction angle & $35^{\circ}$ (medium) or $30^{\circ}$ (weak) \\
\hline Dilation angle & $10^{\circ}$ \\
\hline Peak tensile strength & Variable - from Weibull distribution \\
& Characteristic value \\
& $=4 \mathrm{MPa}$ (medium) or 2.5 MPa (weak) \\
\hline Residual tensile strength & 0 \\
\hline Peak cohesive strength & $2.5 \times$ tensile strength \\
\hline Residual cohesive strength & 0 \\
\hline Elastic boundary block properties & $11 \mathrm{GPa}$ \\
\hline Young's modulus & 0.25 \\
\hline Poison's ratio & \\
\hline
\end{tabular}

For all the simulations with bolt support, the bolt properties, geometry and location remain the same. The bolt configuration is shown in Figure 1. The modelled support design uses cables and grouted rebars. Both types of bolts are represented with the 3DEC hybrid bolt model. All bolts are plated, with the plate assumed to result in a rigid connection with the tunnel surface. The calibrated properties for rebar and cable are presented in Table 2.

Table 2 Hybrid bolt properties calibrated for rebar and cable

\begin{tabular}{lll}
\hline Hybrid bolt properties (3DEC variable name) & Rebar & Cable \\
\hline Cable element Young's modulus (emod) & $1.4 \cdot 10^{11} \mathrm{~Pa}$ & $1.4 \cdot 10^{11} \mathrm{~Pa}$ \\
\hline Node spacing (slen) & $10 \mathrm{~cm}$ & $10 \mathrm{~cm}$ \\
\hline Grout stiffness (kbond) & $3 \cdot 10^{8} \mathrm{~N} / \mathrm{m} / \mathrm{m}$ & $3 \cdot 10^{7} \mathrm{~N} / \mathrm{m} / \mathrm{m}$ \\
\hline Grout strength (sbond) & $2.8 \cdot 10^{5} \mathrm{~N} / \mathrm{m}$ & $2.3 \cdot 10^{5} \mathrm{~N} / \mathrm{m}$ \\
\hline Axial yield strength (yield) & $1.8 \cdot 10^{5} \mathrm{~N}$ & $2.0 \cdot 10^{5} \mathrm{~N}$ \\
\hline Axial rupture strain (strain_limit) & 0.2 & 0.2 \\
\hline Dowel shear stiffness (dowel_stiffness) & $1 \cdot 10^{7} \mathrm{~N} / \mathrm{m}$ & $8 \cdot 10^{6} \mathrm{~N} / \mathrm{m}$ \\
\hline Dowel shear strength (dowel_yield) & $6.3 \cdot 10^{4} \mathrm{~N}$ & $1.9 \cdot 10^{5} \mathrm{~N}$ \\
\hline Shear rupture strain (dowel_strain_limit) & 0.4 & 0.26 \\
\hline
\end{tabular}


When present, surface pressure is modelled as a constant pressure of $100 \mathrm{kPa}$ exerted on the tunnel boundary post-excavation. This pressure might be equivalent to what a well-integrated system of bolts, mesh and shotcrete provide. No pressure is applied on the tunnel floor.

\section{Sensitivity analysis}

\subsection{Simulation sequence}

The tunnel excavation process is simulated and the final state of the excavation ( $100 \%$ deconfined tunnel) is then subjected to a generic caving-induced stress path that simulates a loading/unloading cycle, presented in Figure 2. Only the vertical stress is varied while applied horizontal stresses remain constant.

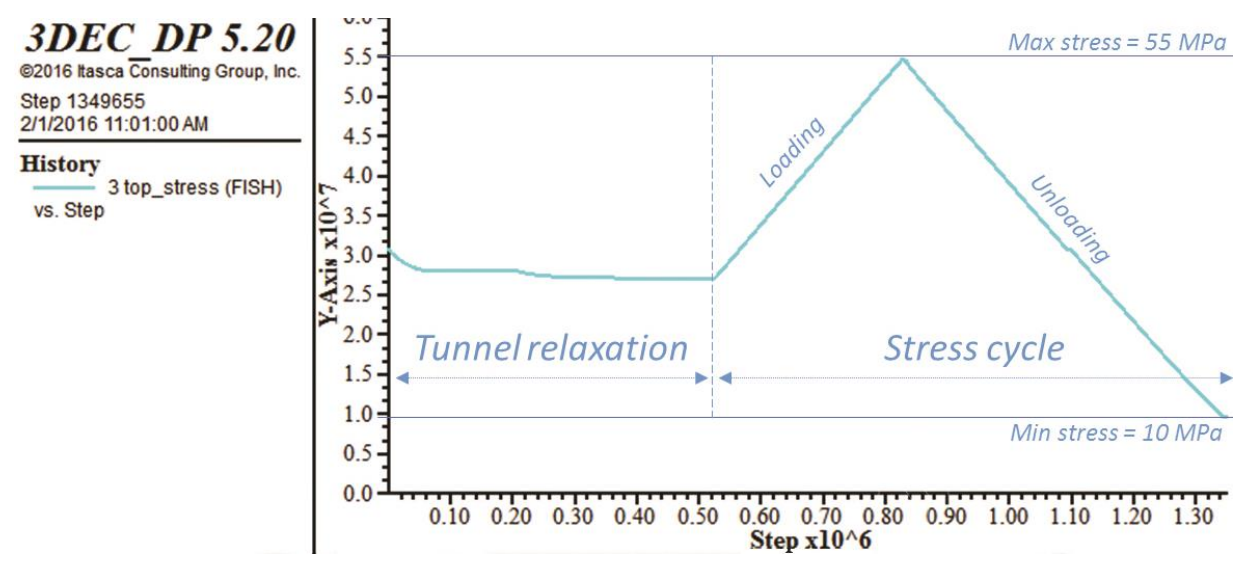

Figure 2 Vertical stress history at the top of the model

The model is $2.5 \mathrm{D}$ in order to permit rapid simulation of the support performance in a sensitivity analysis context where multiple simulations have to be run. Since the BBM is $3 D$, it is possible to simulate a much longer tunnel length in which the actual excavation sequence is modelled. In this $2.5 \mathrm{D}$ test case, the gradual relaxation (i.e. deconfinement) of the tunnel caused by face advance during the excavation is mimicked through application of the 3DEC 'boundary reaction' command to the tunnel boundary. Once the inside of the tunnel is removed, this command calculates the unbalanced forces at the tunnel boundary (equivalent to the in situ stress before starting the relaxation), applies the opposite of those forces to the tunnel boundary, and gradually reduces them to simulate the gradual relaxation of the tunnel. The force decrease is controlled by an exponential law based on the number of cycles performed by 3DEC. Every cycle, the force applied at the tunnel boundary is reduced. Once the forces applied at the tunnel boundary are reduced to $0.5 \%$ of the initial in situ forces, they are removed; the tunnel boundary is free. The model is then cycled until it reaches equilibrium (i.e. until no more significant displacement is observed). After this stage, the tunnel is considered fully relaxed. This last stage (i.e. going from $0.5 \%$ of the in situ stress to $0 \%$ ) can induce a significant amount of displacement, this is discussed in Section 4.1.

The generic stress path imposed on the tunnel is the same for all the simulations. At the end of the tunnel relaxation (i.e. at the end of the excavation), stress is applied at the boundary of the model, based on the following path (see Figure 2):

- Initial vertical stress of $31 \mathrm{MPa}$ corresponding to $1,200 \mathrm{~m}$ depth in situ stress (before tunnel excavation).

- Loading phase: increase the vertical stress until reaching $55 \mathrm{MPa}$.

- Unloading phase:

- 'Partial' unloading: reducing the vertical stress until reaching back to the initial vertical stress of $31 \mathrm{MPa}$.

- Further unloading of the model until reaching $10 \mathrm{MPa}$. 


\subsection{Tested scenarios}

Several cases have been studied in which the support, the support installation sequence and the rock properties, were varied. A total of 11 simulations have been performed, which are listed in Table 3 and described below.

Table 3 List of the simulations to study supported tunnel performance

\begin{tabular}{|c|c|c|c|c|c|c|}
\hline Name & $\begin{array}{l}\text { Rock } \\
\text { tensile } \\
\text { strength } \\
\text { (MPa) }\end{array}$ & $\begin{array}{l}\text { Rock } \\
\text { friction } \\
\text { angle } \\
\left({ }^{\circ}\right)\end{array}$ & Bolt support & $\begin{array}{l}\text { Tunnel } \\
\text { relaxation } \\
\text { at bolts } \\
\text { installation }\end{array}$ & $\begin{array}{l}\text { Surface } \\
\text { pressure } \\
\text { (kPa) }\end{array}$ & $\begin{array}{l}\text { Tunnel } \\
\text { relaxation at } \\
\text { pressure } \\
\text { installation }\end{array}$ \\
\hline \multicolumn{7}{|c|}{ Medium rock mass (reference case) } \\
\hline 01 & 4 & 35 & None & - & None & - \\
\hline 02 & 4 & 35 & $\begin{array}{l}\text { Bonded cables } \\
\text { Grouted rebars }\end{array}$ & $70 \%$ & None & - \\
\hline 03 & 4 & 35 & $\begin{array}{l}\text { Debonded cables } \\
\text { Grouted rebars }\end{array}$ & $70 \%$ & None & - \\
\hline 03-nd & 4 & 35 & $\begin{array}{l}\text { Debonded cables } \\
\text { Grouted rebars } \\
\text { No dowel effect }\end{array}$ & $70 \%$ & None & - \\
\hline \multicolumn{7}{|c|}{ Weak rock mass } \\
\hline W01 & 2.5 & 30 & None & - & None & - \\
\hline W01b & 2.5 & 30 & None & - & 100 & $70 \%$ \\
\hline W02 & 2.5 & 30 & $\begin{array}{l}\text { Bonded cables } \\
\text { Grouted rebars }\end{array}$ & $70 \%$ & 100 & $70 \%$ \\
\hline W02b & 2.5 & 30 & $\begin{array}{l}\text { Bonded cables } \\
\text { Grouted rebars }\end{array}$ & $95 \%$ & 100 & $70 \%$ \\
\hline W03 & 2.5 & 30 & $\begin{array}{l}\text { Debonded cables } \\
\text { Grouted rebars }\end{array}$ & $70 \%$ & 100 & $70 \%$ \\
\hline W03b & 2.5 & 30 & $\begin{array}{l}\text { Debonded cables } \\
\text { Grouted rebars }\end{array}$ & $95 \%$ & 100 & $70 \%$ \\
\hline W03b-nd & 2.5 & 30 & $\begin{array}{l}\text { Debonded cables } \\
\text { Grouted rebars } \\
\text { No dowel effect }\end{array}$ & $95 \%$ & 100 & $70 \%$ \\
\hline
\end{tabular}

In terms of bolt support, the following options have been studied:

- The tunnel is not supported by bolts.

- The tunnel is supported by plated and fully grouted rebars and cables.

- The tunnel is supported by plated and fully grouted rebars and plated and partially debonded cables.

A first series of simulations has been performed on the medium rock mass. It led to a horizontal wall displacement around $6 \mathrm{~cm}$ at the end of the loading cycle when supported. For this set of simulations, bolts have been installed at $70 \%$ of relaxation, which corresponds to installing the bolts about half a tunnel 
radius ( $1 \mathrm{~m}$ ) behind the face (Vlachopoulos \& Diederichs 2009). In order to observe support effectiveness in a more dramatic scenario, in terms of rock quality, the rock mass properties have been reduced from 'medium' to 'weak' rock mass properties (see Table 3 for detailed properties).

The weak rock mass leads to significantly more bulking and damage around the tunnel. Because the rock was so weak, a small surface pressure had to be applied on the tunnel boundary to avoid collapse of the fractured 'skin'. For this weak rock mass, the displacement/relaxation curve is quite different than for the medium rock mass. Most of the tunnel wall displacement happens after $90 \%$ of relaxation. Therefore, installing the bolts at $70 \%$ of relaxation corresponds to a very early stage (less than half a tunnel radius behind the face) and leads to bolt failure before the end of the relaxation. To address this issue, an additional series of runs were performed to see if a later installation would improve support effectiveness. In these additional runs, the bolts are installed at $95 \%$ of relaxation, which corresponds to an installation about one tunnel radius behind the face $(\sim 2.2 \mathrm{~m})$.

For both types of rock mass, simulations have been run without any support, as well as with only a small surface pressure of $100 \mathrm{kPa}$ in the case of the weaker rock mass. The surface pressure is installed at $70 \%$ of tunnel relaxation. For both types of rock mass, simulations have been run with rebars and cables grouted on their whole length. They are referred to as 'bonded cable' simulations. Equivalent simulations have been run where only cables (not rebars) are not grouted along the first half length (the half section closest to the tunnel wall). These are referred to as 'debonded cable' simulations. In practice, the 'debonding' of a cable section is realised by zeroing the grout strength along the section. For some cases, the effect of the dowel resistance modelled with the hybrid bolts has been assessed by running an equivalent simulation without dowel resistance.

\section{$4 \quad$ Results}

\subsection{Effect of support on bulking and damage}

This section explores the effect that surface pressure and bolts have on the damage and bulking evolution adjacent to the excavation.

Figure 3 presents contours of displacement for the weak rock mass at various stages of the simulation: at the end of the excavation (i.e. the tunnel is fully relaxed), when the tunnel is partially loaded, at the end of the loading phase and finally at the end of the unloading phase. Three cases are studied here: unsupported tunnel, surface pressure of $100 \mathrm{kPa}$, and tunnel supported by both pressure and bolts (with debonded cables and bolts installed at $95 \%$ of tunnel relaxation). This figure also shows fracture opening greater than $0.8 \mathrm{~mm}$ (identified as black lines), which is used as a criterion to define the approximate inner shell limit. The inner shell limit is defined as the limit of the volume of rock where fractures opening more than $0.8 \mathrm{~mm}$ are significant. Note that the $0.8 \mathrm{~mm}$ criterion is only used to compare different cases; this is not an absolute criterion. Changing the criterion would yield different inner shell dimensions.

Figure 3 shows that the tunnel performance is very different when unsupported as compared to a tunnel supported by bolts or even by only $100 \mathrm{kPa}$ of surface pressure. When $100 \mathrm{kPa}$ of surface pressure is applied, bulking of the tunnel is significantly reduced and collapse of the roof is prevented. For the unsupported case, the inner shell tends to grow at the beginning of the loading. Its limit remains fairly stable during the rest of the loading phase. The unloading process causes minor additional bulking in the 'highly bulked' region, but it deepens the bulking zone. Thus, bulking of the newly fractured rock mass in the expanding inner shell 'pushes' the previously bulked material into the excavation. The presence of surface pressure limits the growth of the inner shell during the loading phase. It also reduces the formation and opening of fractures inside the inner shell. During the unloading phase the inner shell tends to grow, but it remains significantly smaller than in the unsupported case. The intensity of the bulking is also significantly smaller than in the unsupported case. When the tunnel is supported by pressure and bolts, the dimension of the inner shell is slightly smaller but similar to the case where only surface pressure is applied. However, the amount of open fractures and the bulking intensity is significantly reduced. Only for this case, 
the simulation has been run until the end of the unloading. It can be seen that when the tunnel is further unloaded the inner shell dimension is fairly stable, but a lot of new fractures are formed inside the inner shell. The associated bulking increases, but not dramatically.

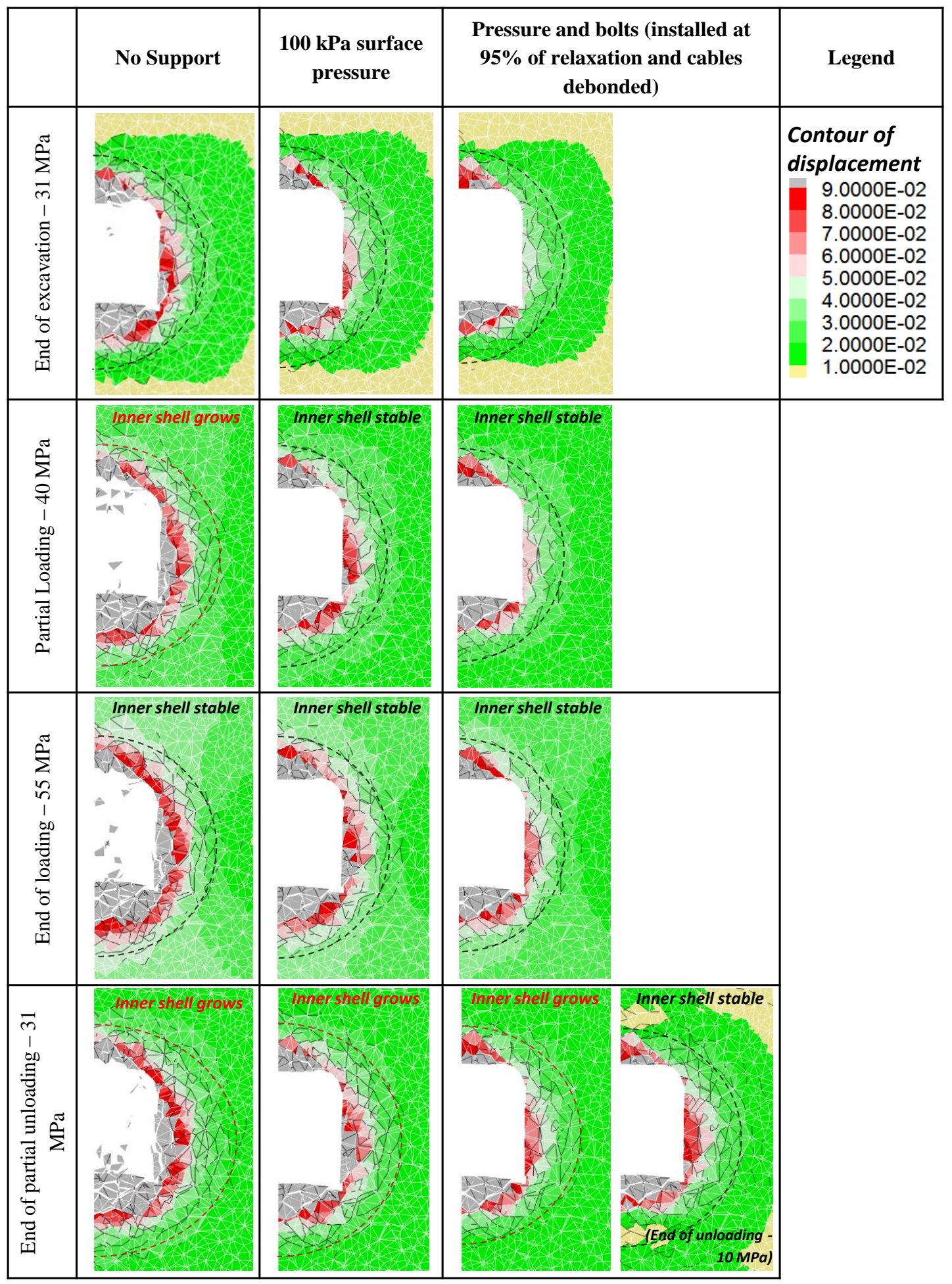

Figure 3 Effect of loading cycle on bulking and damage in a weak rock mass when tunnel is unsupported or supported: the colour scale gives the intensity of the displacement and black lines corresponds to fractures open more than $8 \mathrm{~mm}$

Figure 4 shows histories of horizontal displacement in the wall of the tunnel, with and without surface pressure, for a weak rock mass during the excavation only. Displacements are recorded $30 \mathrm{~cm}$ and $1.3 \mathrm{~m}$ 
inside the wall. Figure 5 shows equivalent histories for the excavation, the loading, and the partial unloading. In Figure 4, it can be seen that during the relaxation of the unsupported tunnel, the wall cumulative displacement significantly increases from $3.6 \mathrm{~cm}$, when the applied load on the tunnel boundary is still $0.5 \%$ of the initial in situ stress ( $450 \mathrm{KPa}$ normal pressure on the wall), to $6.4 \mathrm{~cm}$ after the remaining load has been removed. This indicates that any pressure applied on the tunnel boundary, even very small, significantly limits the formation of tensile and shear fractures and the associated bulking of the damaged rock. The same conclusion appears when comparing the final displacement at the end of the relaxation of the unsupported tunnel and the tunnel where a $100 \mathrm{kPa}$ surface pressure is applied. Applying this small amount of pressure reduces the final horizontal wall displacement by $25 \%$. The displacement reduces from $6.4 \mathrm{~cm}$ (for the unsupported case) to $4.8 \mathrm{~cm}$ (for the case with surface pressure). As shown in Figure 3, the small amount of pressure applied at the tunnel boundary prevents the bulking of the wall and the roof, as well as preventing roof fragments from collapsing into the tunnel, during the whole loading cycle.

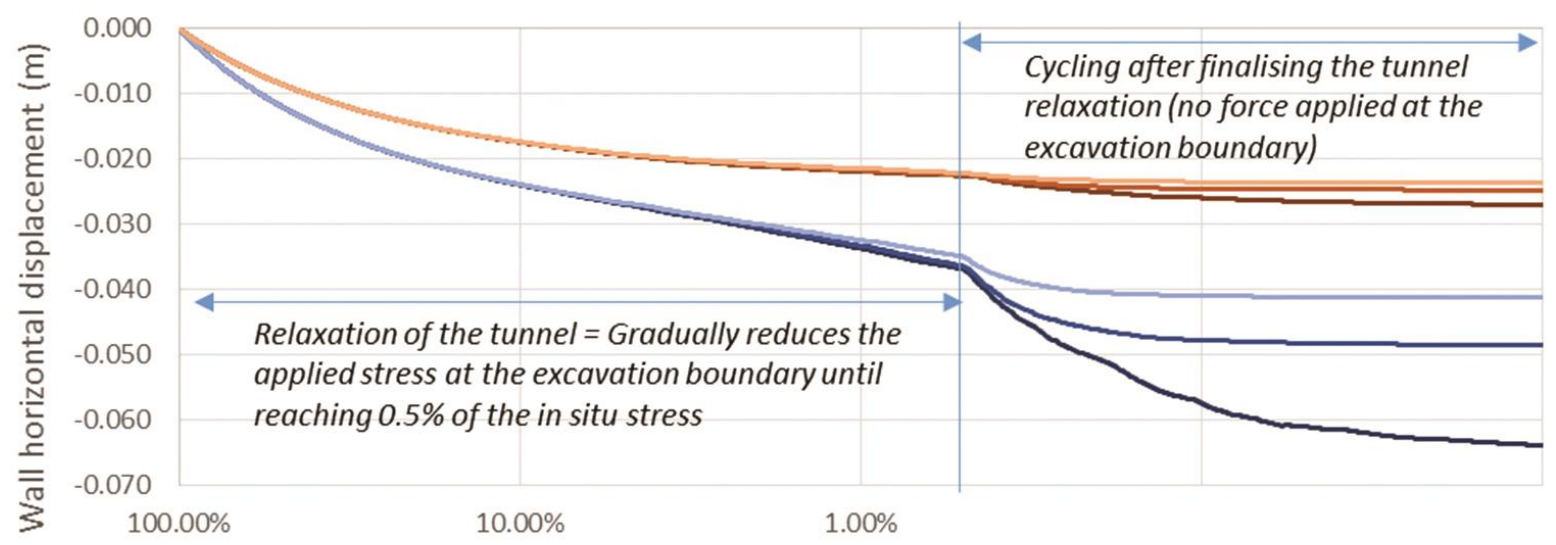

Ratio current stress/in situ stress
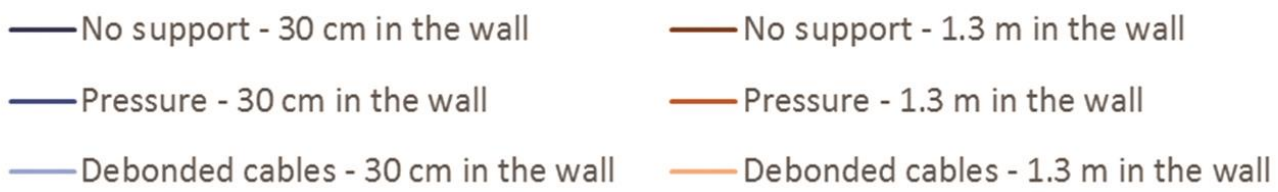

Figure 4 Histories of horizontal displacement in the wall of the tunnel, without and with support, for a weak rock mass during the excavation of the tunnel
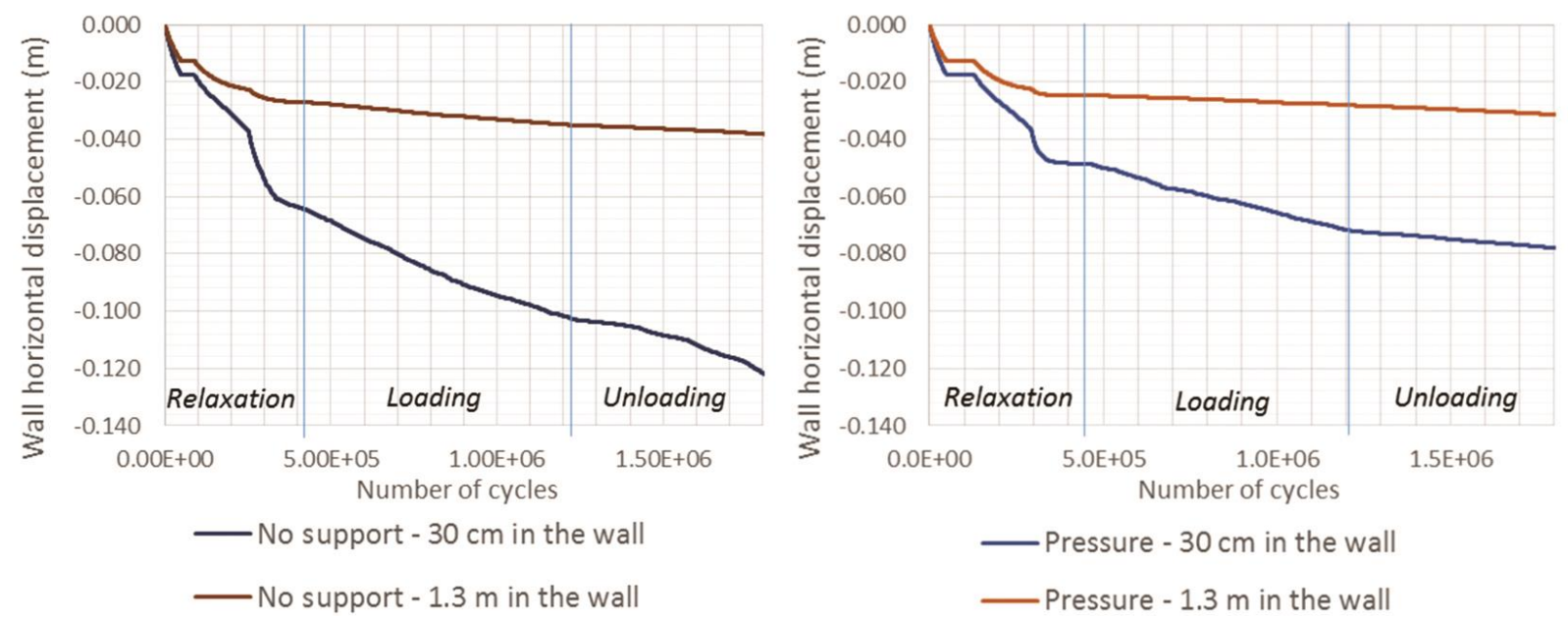

Figure 5 Histories of horizontal displacement in the wall of the tunnel: (left) without support, and (right) with $100 \mathrm{kPa}$ surface pressure, for a weak rock mass during the excavation, the loading, and the partial unloading of the tunnel 
Figure 5 compares similar histories of horizontal displacement in the wall of the tunnel as Figure 4 with the loading and partial unloading of the tunnel included. At the end of the partial unloading, the final horizontal displacement drops from $12.3 \mathrm{~cm}$ for the unsupported tunnel to $7.8 \mathrm{~cm}$ when $100 \mathrm{kPa}$ of surface pressure is applied; which corresponds to a reduction of $35 \%$ due to the presence of the surface pressure. It can also be observed that when surface pressure is present, the velocity (i.e. increase of displacement with unloading) of the wall decreases during the unloading phase while it stays constant when the tunnel is completely unsupported.

This behaviour was well explained by Garza-Cruz and Pierce (2014). As loading of the tunnel model increases, less fracturing occurs in the vicinity of the tunnel when surface pressure is applied because of the confinement effect induced near the excavation. This increase of confinement inhibits the formation of tensile and shear fractures suppressing bulking of the damaged rock mass in the tunnel periphery. This behaviour contrasts with the unsupported tunnel whose unconfined walls are able to fracture more and continue to bulk at a high rate, due to a geometrical bulking effect of non-fitting blocks. The applied surface pressure also reduces the rate of confinement loss when the tunnel is unloaded, diminishing fracture initiation and propagation and, so, reducing the ability of the inner-shell to grow. Thus, additional unidirectional bulking is also very limited because the main contribution during the unloading is from bulking of the newly fractured rock mass in the expanding inner shell, which 'pushes' the previously bulked material into the excavation.

In conclusion, these models show that:

- Even $100 \mathrm{kPa}$ of surface pressure significantly reduces dimensions of the inner shell (fractured region) and the degree of bulking during the excavation but, also during the loading cycle, reaffirming the large effect of confinement on rock mass strength.

- Even $100 \mathrm{kPa}$ of surface pressure prevents collapse of the roof.

- The presence of bolts does not significantly reduce the dimensions of the inner shell when compared to the case where only surface pressure is applied, but it limits the formation and opening of fractures inside the inner shell, thereby, limiting the magnitude of the wall horizontal displacement.

\subsection{Influence of debonding the cables and installation sequence on support effectiveness}

This section explores the effect that cable debonding and bolt installation timing have on the bulking evolution adjacent to the excavation. The effect on the damage of the cables themselves is also investigated.

Figure 6 shows bolts axial force and axial strain at the end of the unloading for the medium rock mass and the weak rock mass when supported by bonded cables and debonded cables. Figure 7 shows zoomed views of Figure 6 of the second lowest cable to illustrate in details the change in behaviour depending on the rock mass quality and the type of installation. Figure 8 shows corresponding contours of displacement. The plots in Figure 6 and Figure 7 have four components of information: (i) axial force is shown as a perpendicular bar chart, (ii) axial strain is shown as a colour contour along the support, (iii) support axial yield and rupture are shown as colour indicators below the support, and (iv) displacement in the rock is shown as greyscale block contour. 


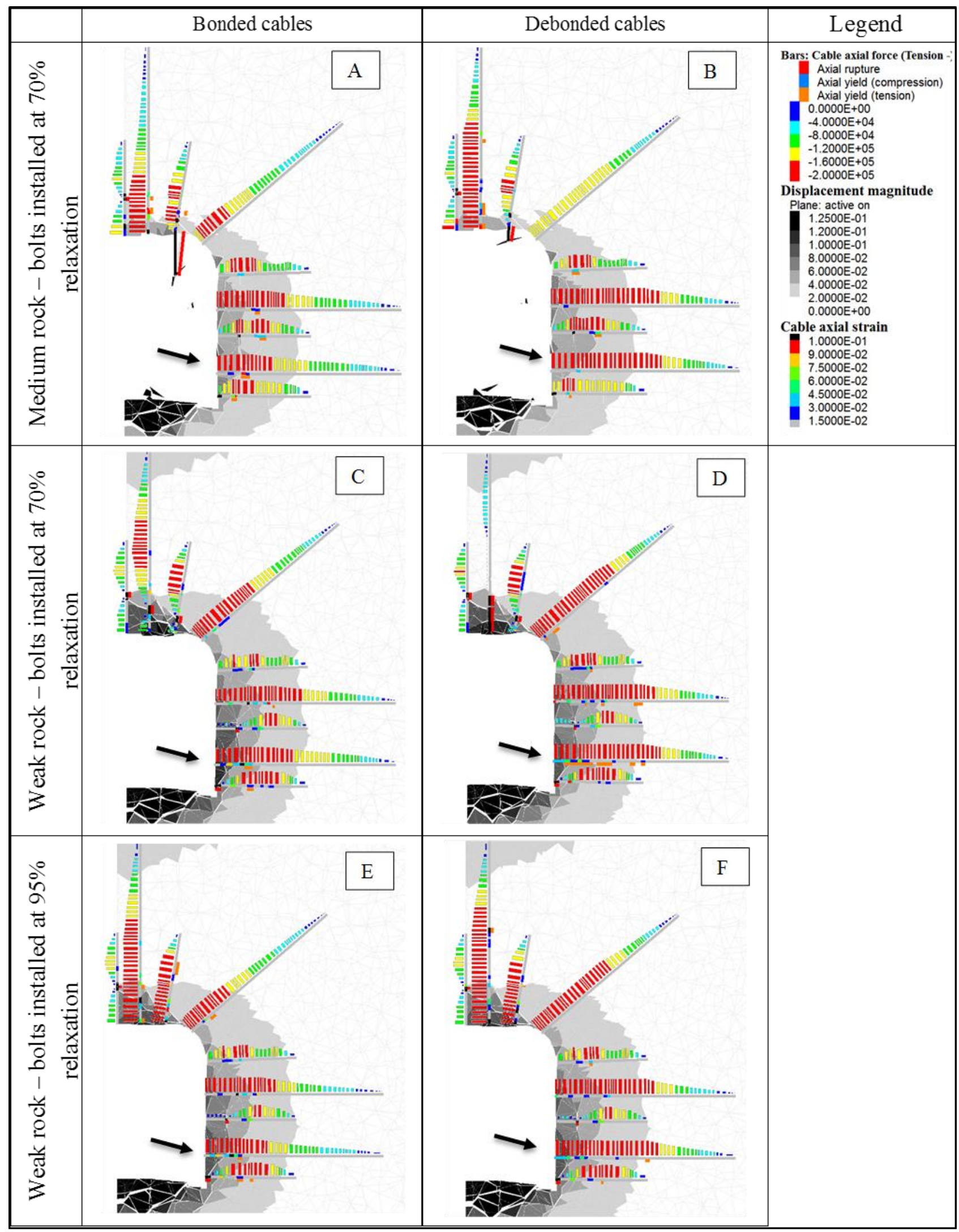

Figure 6 Axial force and axial strain of supports at the end of unloading (long supports are cables and short supports are grouted rebars) 


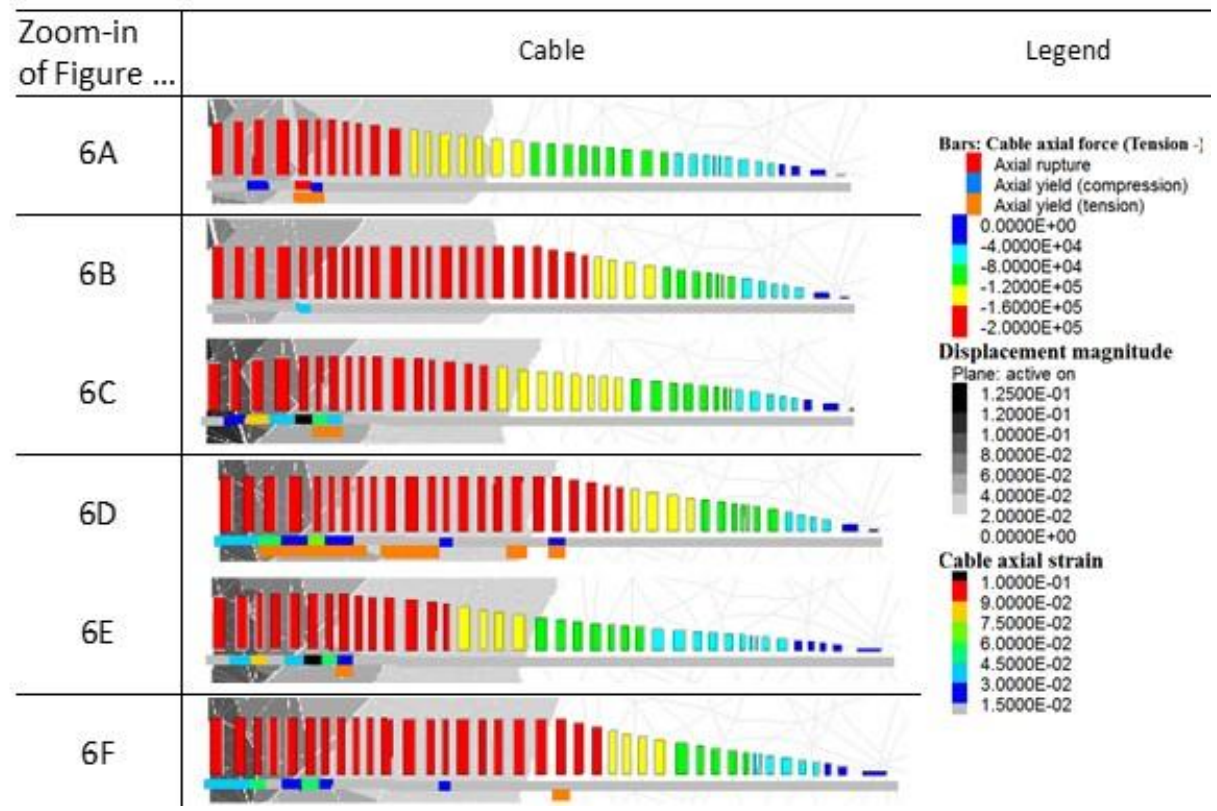

Figure 7 Axial force and axial strain at the end of unloading. Zoom-in of Figure 6 of the second lowest cable

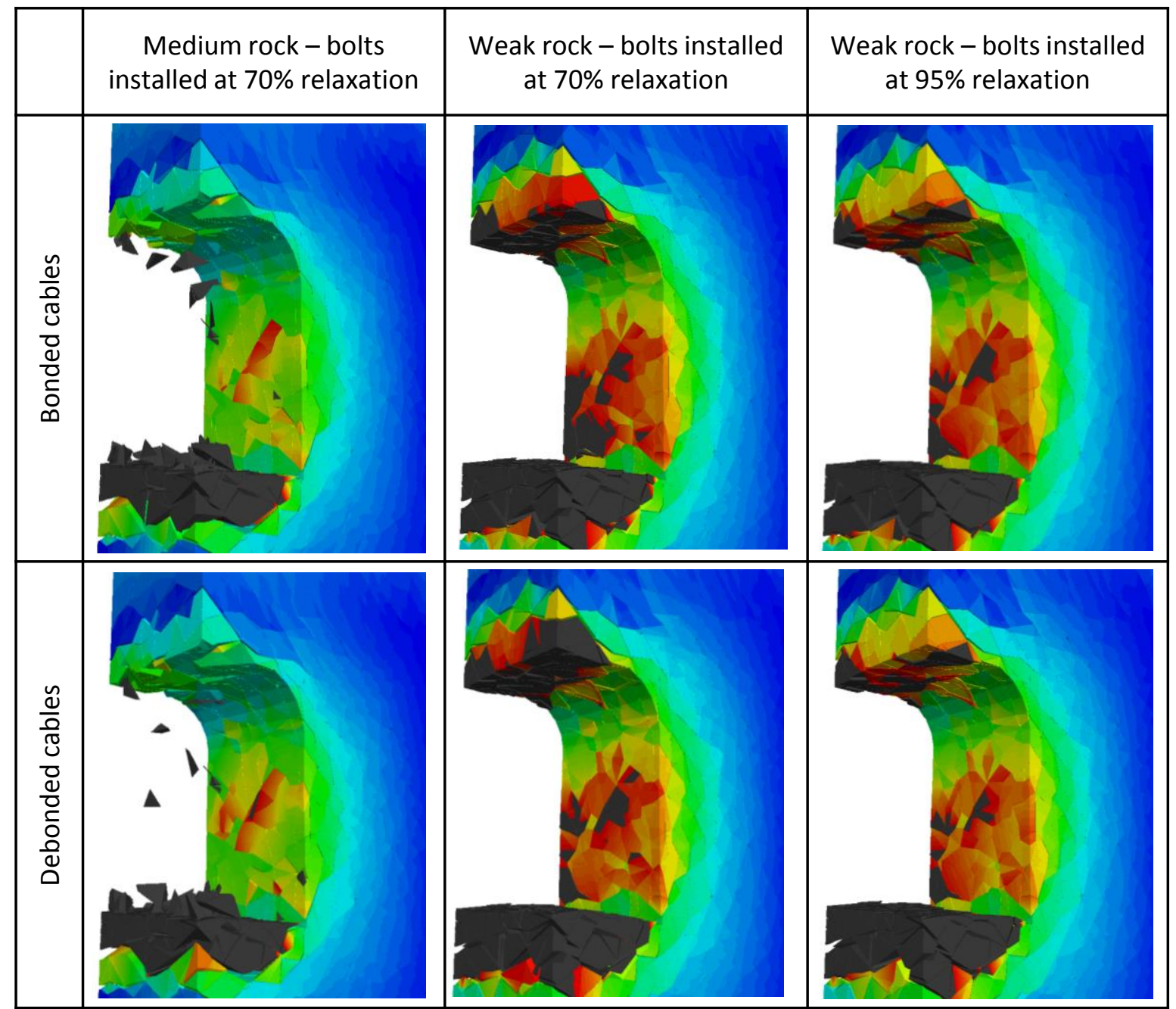

Figure 8 Contour of displacement at the end of unloading for the medium rock mass and the weak rock mass 
As shown in Figure 8, when installed in a medium quality rock mass, support with bonded cables is slightly more efficient in terms of final bulking of the tunnel wall. For a lower quality rock mass, the debonded cables give better results when they are installed very early (at $70 \%$ of relaxation, or $1 \mathrm{~m}$ behind the face). This is also true when they are installed further behind the face (at $95 \%$ of relaxation or $2 \mathrm{~m}$ behind the face), but less pronounced.

Indeed, as seen in Figure 6, when cables are debonded along half their length, any axial displacement on this section of the cable is spread over the entire debonded section. This leads to more uniform and lower axial strain and maximum force along the debonded section, which can prevent axial yielding or failure. However, this reduces the effectiveness of the cable locally. When the cable is bonded, the deformation is more local and the local force increase is higher. This can be seen on the cable pointed out, by the arrow, in Figures 6(e) and 6(f), (zoomed views are available in Figure 7). When the cables are debonded (Figure 6(f)), the axial force is uniform along the debonded section. The maximum axial strain is around $6 \%$, and there is no axial yield of the cable along the debonded section. When the cable is bonded (Figure 6(e)), the axial force is not uniform and is related to the local rock deformation. The maximum axial strain is above $10 \%$ (black section of the cable), and axial yielding can be observed (orange indicator below the cable). However, in the same cable on Figures 6(c) and 6(d), when the bolts are installed at an earlier stage of the relaxation, this observation is no longer true. In Figure $6(d)$ it can be seen that the axial displacement of the cable is enough that, although it is spread on the debonded section, axial yielding still occurs. Because the section is debonded, nearly the entire debonded section is at, or near, the maximum bolt capacity. If the cable is bonded, yielding is also observed, but it is much more localised.

The following conclusions are drawn:

- When a section of the cable is debonded, it spreads the cable strain along the debonded section, which can prevent, in some cases, cable failure. But this also reduces the bolt's effectiveness locally.

- In the case of excessive deformation when the cable fails, the debonded section can no longer carry load. This is not true for a bonded cable.

- In case of good rock mass conditions where cable failure is unlikely to happen, bonded cables appear to be more efficient.

In case of poor rock mass conditions, when large deformation of the rock mass is expected, debonded cable can be more efficient than bonded ones; since debonding can prevent cable failure in some cases. In those conditions, failure can still happen. Installing the bolts at a later stage of the excavation can help prevent bolt failure.

\subsection{Influence of the bolt shear resistance on support effectiveness}

As explained in detail in Bouzeran et al. (2016), 3DEC hybrid bolt elements are an update to 3DEC cable elements to better represent shear resistance of rockbolts, the so-called 'dowel effect'. This section explores the dowel effect on the damage and bulking evolution adjacent to the excavation.

Figure 9 shows horizontal displacement at the end of the loading cycle, when the tunnel is supported with debonded cables for a medium and a weak quality rock masses. For each case, in addition to the default simulation (for which bolts resist in both the axial and shear directions), an equivalent simulation has been run where the dowel effect of bolts is disabled (i.e. the bolts' shear resistance is not modelled).

It can be seen in Figure 9 that, while the dowel effect is present, the wall bulking is not significant for a medium quality rock, it is important for weaker rock. Indeed, as shown in Figure 10 for the medium quality rock, the amount of shear displacement along the bolts is fairly small for most bolts, with a maximum value of around $2 \mathrm{~cm}$. In consequence, the shear resistance provided by the bolts is also small (mostly below $80 \mathrm{kN}$ ). Note that the maximum shear resistance of bolts modelled in these simulations is around $200 \mathrm{kN}$. For the weaker rock, shear displacement above $2.5 \mathrm{~cm}$ can be observed in several locations along the bolts. The bolts provide shear resistance, above $100 \mathrm{kN}$ in several locations. The location of maximum bolt shear resistance occurs in the roof and is circled in red. 


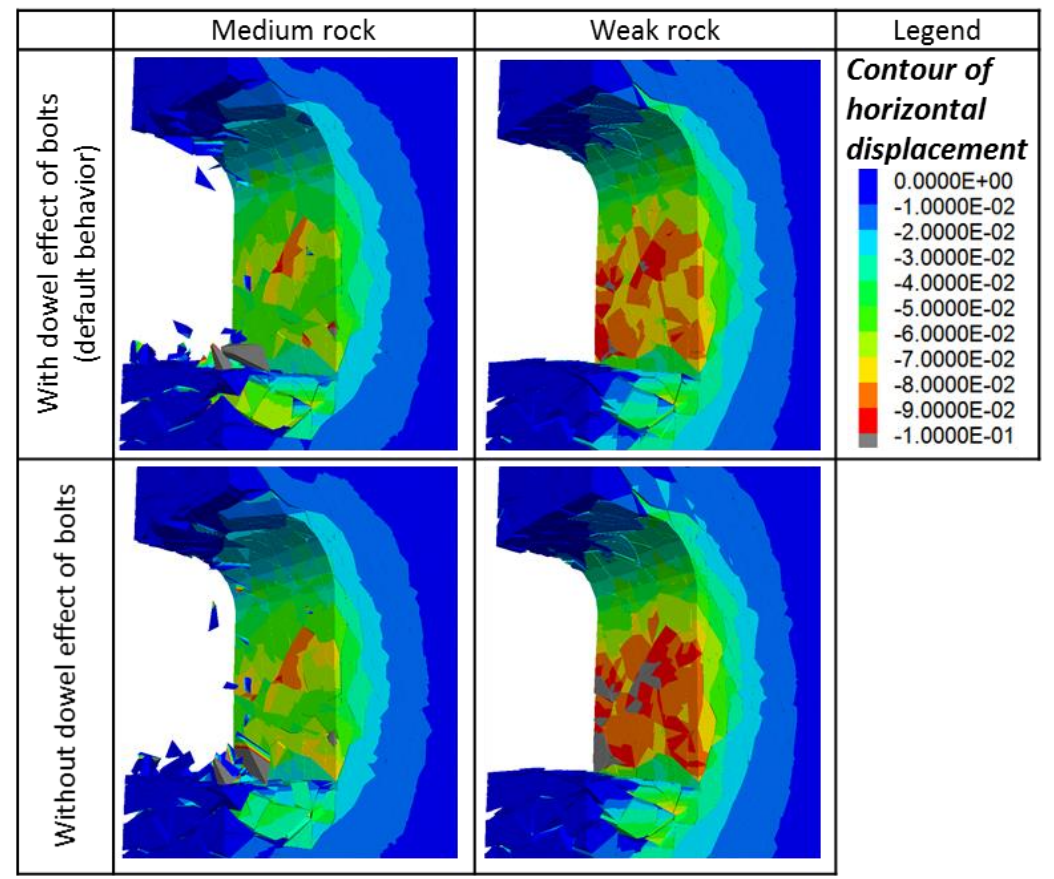

Figure 9 Horizontal displacement of the tunnel wall at the end of partial tunnel unloading for a medium rock mass and a weak rock mass with bolts support (cables are debonded)

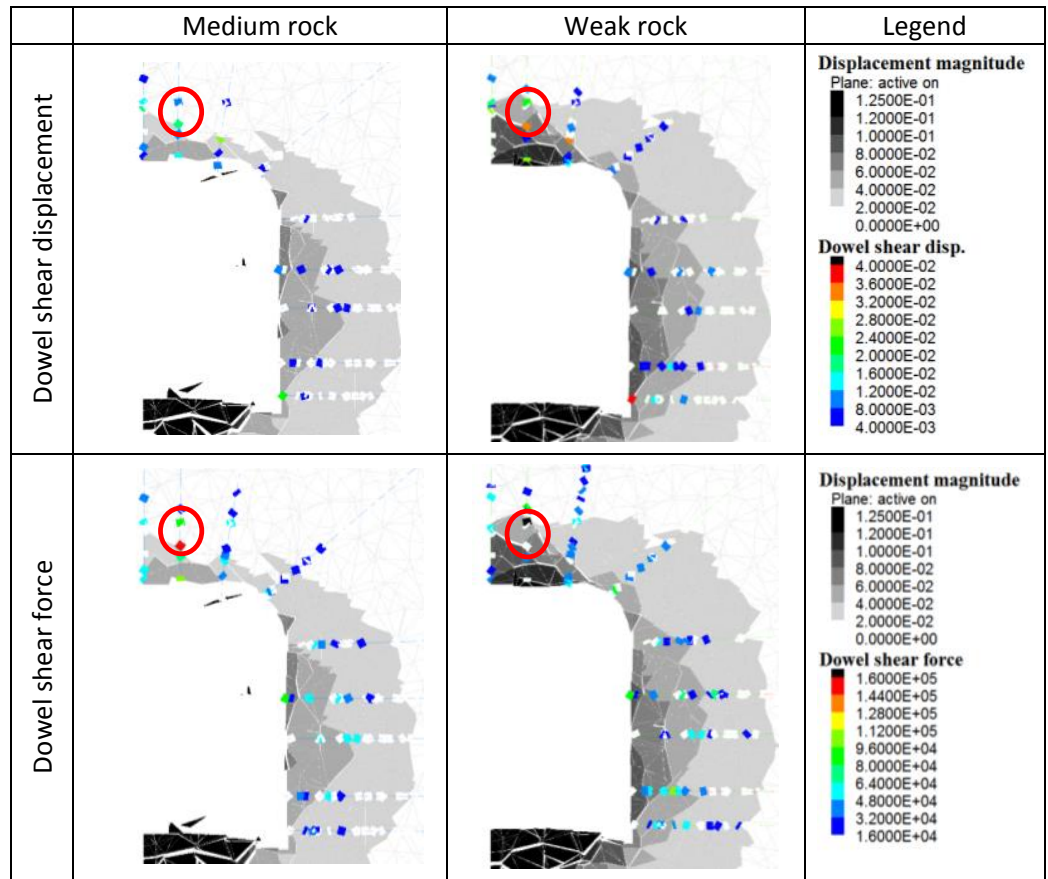

Figure 10 Dowel shear displacement and force at the end of unloading for a medium and a weak rock and debonded cables

The conclusions are:

- Dowel action can have significant impact on tunnel performance.

- The weaker the rock mass, i.e where more fractures are likely to be formed and exhibit significant opening and shearing, the more effective the bolt dowel action. 


\section{Conclusion}

A BBM tunnel model has been built to study support effectiveness with tunnelling at depth. A real-life design has been studied involving cables and grouted rebars - both modelled with calibrated 3DEC hybrid bolts. A $100 \mathrm{kPa}$ surface pressure has also been installed in some cases. The models have examined the effect that bolts and surface pressure have on the damage development and bulking evolution adjacent to the excavation during tunnel loading/relaxing due to a cave-like stress cycle. Different behaviours have been observed when support is installed in different qualities of rock. More precisely, the effect of bonding versus debonding the cables has been explored. The comparison of these models has led to the following main observations.

- Even $100 \mathrm{kPa}$ of surface pressure significantly reduces fracture formation and the degree of bulking around the tunnel during the excavation and the loading cycle. This reaffirms the large effect of confinement on rock mass strength.

- When a section of the cable is debonded, it spreads the cable strain along the debonded section. Debonding can prevent cable failure in some cases. However, debonding reduces the local effectiveness of the bolt.

- In the case of excessive deformation, if the cable fails, the debonded section can no longer carry load, which is not true for a bonded cable.

- In good rock mass conditions, where cable failure is unlikely to happen, bonded cables appear to be more efficient.

- In poor rock mass conditions, when large deformation of the rock mass is expected, debonded cables can be more efficient than bonded ones; since debonding can prevent cable failure in some cases. In these conditions, failure can still happen. Installing the bolts at a later stage of the excavation can help prevent failure.

- The bolt dowel action can have significant impact on tunnel performance. The weaker the rock mass and the larger the deformation, the more this is true.

The bolt behaviour observed in the example models seems realistic and in accordance with field observations. The next phase of this ongoing development is to further test and validate the BBM models and associated support elements. The ultimate goal is to use this model as an aid to design and complement existing empirical approaches when facing particularly challenging rock conditions (e.g. high stress to strength ratios and/or complex stress changes).

\section{Acknowledgement}

The authors acknowledge the Rio Tinto Centre for Underground Mine Construction for partial funding of the work presented in this paper.

\section{References}

Bouzeran, L, Furtney, J, Hazzard, J, Lemos, JV \& Pierce, M 2016, 'Advanced 3DEC bolt model for simulation of ground support performance in highly fractured and bulked rock masses', in E Nordlund, TH Jones \& A Eitzenberger (eds), Proceedings of the Eighth International Symposium on Ground Support in Mining and Underground Construction, 12-14 September, Luleå, Sweden, Luleå University of Technology, paper 210, pp. 11.

Garza-Cruz, T \& Pierce, M 2014, 'A 3DEC Model for Heavily Veined Massive Rock Masses', Proceedings of the 48th US Rock Mechanics/Geomechanics Symposium, American Rock Mechanics Association, Alexandria.

Itasca Consulting Group, Inc. 2013, 3DEC - Three-Dimensional Distinct Element Code, software, version 5.0, Minneapolis.

Vlachopoulos, N \& Diederichs, MS 2009, 'Improved Longitudinal Displacement Profiles for Convergence Confinement Analysis of Deep Tunnels', Rock Mechanics and Rock Engineering, vol. 42, no. 2, pp. 131-146. 